\title{
Subloop Deformation Behavior of TiNi Shape Memory Alloy Subjected to Stress-Controlled Loadings
}

\author{
Elzbieta A. Pieczyska ${ }^{1}$, Hisaaki Tobushi ${ }^{2, * 1}$, Wojciech K. Nowacki ${ }^{1}$, \\ Stefan P. Gadaj ${ }^{1}$ and Toshimi Sakuragi ${ }^{2, * 2}$ \\ ${ }^{1}$ Institute of Fundamental Technological Research, Polish Academy of Sciences, Swietokrzyska 21, Warsaw, 00-049, Poland \\ ${ }^{2}$ Department of Mechanical Engineering, Aichi Institute of Technology, Toyota 470-0392, Japan
}

\begin{abstract}
The main characteristics which appear in shape memory alloys (SMAs) are the shape memory effect and superelasticity. In applications of SMAs, the thermomechanical properties of SMAs are most important. The return-point memory does not appear under the stress-controlled conditions. Creep and stress relaxation can be induced due to the phase transformation in the subloop loading under the stress-controlled conditions. In order to design the SMA elements properly, it is important to understand the influence of the thermomechanical loading conditions on the nucleation and progress of the phase transformation and the corresponding deformation behaviors. In the present paper, the conditions for the nucleation and progress of the phase transformation are investigated for SMAs subjected to the subloop loadings under the stress-controlled conditions. The uniaxial tension tests for the TiNi SMAs were carried out in the superelastic region under the various thermomechanical loading conditions. The thermomechanical conditions for the progress of the phase transformation are discussed in the subloop loading under the stresscontrolled conditions. Strain increases during unloading and decreases during reloading under the stress-controlled subloop loading. These pseudoviscoelastic behaviors are important for the precise control of SMA elements. [doi:10.2320/matertrans.MRA2007097]
\end{abstract}

(Received May 1, 2007; Accepted July 13, 2007; Published September 25, 2007)

Keywords: shape-memory alloy, titanium-nickel alloy, subloop, superelasticity, creep, stress relaxation, neutral loading

\section{Introduction}

The main characteristics which appear in shape memory alloys (SMAs) are the shape memory effect (SME) and superelasticity (SE) ${ }^{1-4)}$ Large recovery strain, high recovery stress, large energy storage and high damping can be obtained due to these properties by SMAs. SMAs have such excellent properties and therefore their developments and applications are expected as the smart materials and structures. In applications of SMAs, the thermomechanical properties of SMAs are most important since the driving force and the working stroke vary depending on the variation in temperature and they must be controlled precisely. If temperature varies above and below the transformation finish temperatures, the thermomechanical condition is called the perfect loop or full loop, and the expected driving force and working stroke can be obtained. However, if temperature varies between the transformation start and finish temperatures (this case is called the subloop or internal loop), the expected values of the driving force and the working stroke can not be obtained. This point is very important from the viewpoint of the precise control of SMA elements. The investigations on the deformation properties performed till now are mainly for the full loop loading under the straincontrolled conditions. ${ }^{5,6)}$ In the case of the subloop loading under a low strain rate, the reloading curve of the stress-strain curve passes through the point where unloading started. ${ }^{7)}$ This property is called as the return-point memory. The return-point memory does not appear under the stresscontrolled conditions. ${ }^{89}$ In applications of SMAs, SMA elements are subjected to various thermomechanical loadings. In order to design the SMA elements properly, it is important to understand the influence of the thermomechan-

\footnotetext{
${ }^{* 1}$ Corresponding author, E-mail: tobushi@aitech.ac.jp

${ }^{* 2}$ Graduate Student, Aichi Institute of Technology
}

ical loading conditions on the progress of the phase transformation and the corresponding deformation behaviors.

In the present paper, the conditions for the nucleation and progress of the phase transformation are investigated in SMAs subjected to the subloop loading under the stresscontrolled conditions. The uniaxial tension tests for TiNi SMAs were carried out in the region of SE under the various thermomechanical loading conditions. The conditions for the progress of the phase transformation and the neutral loading are discussed. Strain increases during unloading and decreases during reloading under the stress-controlled subloop loading. These pseudoviscoelastic behaviors are important for the precise control of SMA elements in applications.

\section{Experimental Method}

\subsection{Materials and specimen}

The materials used in the experiment were a rectilinear Ti55.4 mass $\% \mathrm{Ni}$ SMA wire and a flat Ti-55.3 mass $\% \mathrm{Ni}$ SMA tape which were polycrystalline and produced by Furukawa Electric Co. Diameter of the wire was $0.75 \mathrm{~mm}$, and width and thickness of the tape were $10 \mathrm{~mm}$ and $0.4 \mathrm{~mm}$, respectively. The reverse transformation finish temperatures $A_{f}$ were $323 \mathrm{~K}$ for the wire and $283 \mathrm{~K}$ for the tape, respectively. The displacement was measured by the extensometers with the gauge length $20 \mathrm{~mm}$ and $100 \mathrm{~mm}$ for the wire and the tape, respectively. The average temperature of the tape was measured over an area of $8 \times 60 \mathrm{~mm}$, located in the central part of the specimen by the infrared camera.

\subsection{Experimental procedure}

In order to investigate the deformation properties of the material under the various loading conditions, the following six kinds of thermomechanical tension tests were carried out in air. In the tests except for Exps. V and VI, the ambient temperature was kept constant at $T=353 \mathrm{~K}$. In the Exps. V 


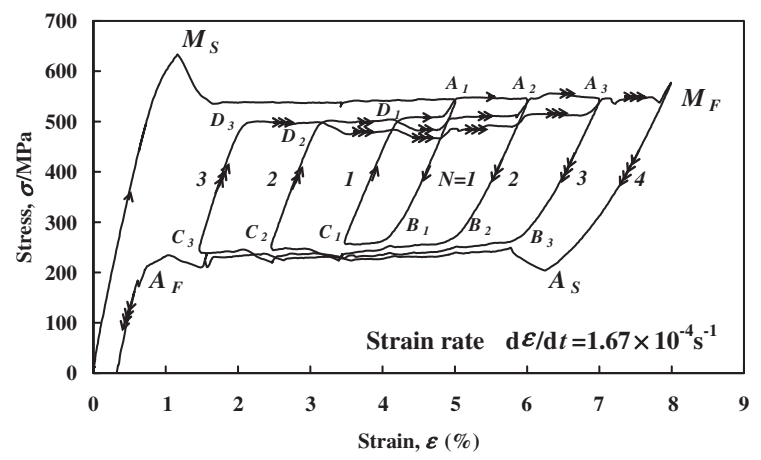

Fig. 1 Stress-strain curves for subloop loading under constant strain rate.

and VI, the ambient temperature was room temperature and was not controlled. Stress and strain were treated in terms of nominal stress and nominal strain, respectively. Therefore the stress-controlled and strain-controlled conditions mean the load-controlled and displacement-controlled conditions, respectively.

Exp. I: Subloop deformation under low strain rate

In the transformation region during loading and unloading, strain was controlled to decrease or increase repeatedly under low strain rate for the wire.

Exp. II: Subloop deformation under various stress rates In the transformation region during loading and unloading, stress was controlled to decrease or increase repeatedly under various stress rates for the wire.

Exp. III: Subloop loading with constant stress

In the transformation region during loading and unloading under constant stress rate, stress was kept constant and variation in strain was observed for the wire.

Exp. IV: Subloop loading with constant strain

In the transformation region during loading and unloading under constant stress rate, strain was kept constant and variation in stress was observed for the wire.

Exp. V: Deformation and temperature variation under various strain rates

Stress-strain relationship and variation in temperature were observed under various strain rates for the tape.

Exp. VI: Neutral loading

In the transformation region during loading, subloop deformation and variation in temperature were observed under the special stress rate for the tape in order to confirm the neutral loading condition.

\section{Subloop Deformation under Various Loading Rates}

\subsection{Subloop deformation under low strain rates}

The stress-strain curves obtained by the subloop loading test under a low modulus of the strain rate $\mathrm{d} \varepsilon / \mathrm{d} t=$ $1.67 \times 10^{-4} \mathrm{~s}^{-1}$ (Exp. I) are shown in Fig. 1. In the test, the process $\left(\mathrm{A}_{i}, \mathrm{~B}_{i}\right.$ and $\left.\mathrm{C}_{i}\right)$ corresponds to unloading and the

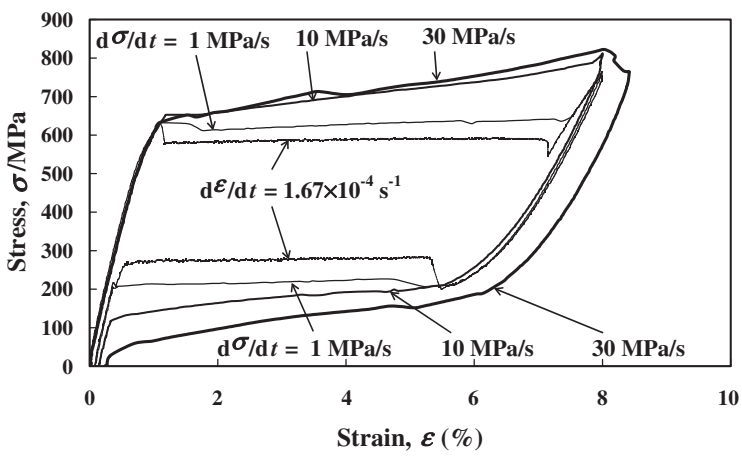

Fig. 2 Stress-strain curves for full loop loading under various stress rates.

process $\left(\mathrm{C}_{i}, \mathrm{D}_{i}\right.$ and $\left.\mathrm{A}_{i+1}\right)$ corresponds to reloading. The process $\left(\mathrm{A}_{i}, \mathrm{~B}_{i}\right)$ and the process $\left(\mathrm{C}_{i}, \mathrm{D}_{i}\right)$ are elastic. The reverse transformation appears in the process $\left(\mathrm{B}_{i}, \mathrm{C}_{i}\right)$ and the MT appears in the process $\left(\mathrm{D}_{i}, \mathrm{~A}_{i+1}\right)$. The MT stress decreases under cyclic deformation. ${ }^{10)}$ Therefore, the MT stress plateau during the reloading process $\left(\mathrm{D}_{i}, \mathrm{~A}_{i+1}\right)$ decreases with increase in the number of cycles $N$. The reloading curve $\left(\mathrm{C}_{i}, \mathrm{D}_{i}\right.$ and $\left.\mathrm{A}_{i+1}\right)$ passes through the unloading start point $\mathrm{A}_{i}$. Therefore, the return-point memory is observed in the case of the strain-controlled condition with a low strain rate.

\subsection{Subloop deformation under various stress rates 3.2.1 Full loop deformation under various stress rates}

The stress-strain curves obtained by the full loop loading under various stress rates for the SMA wire at $T=353 \mathrm{~K}$ are shown in Fig. 2. The stress-strain curve obtained under a strain rate of $1.67 \times 10^{-4} \mathrm{~s}^{-1}$ is also shown in Fig. 2. This strain rate is low enough corresponding to the isothermal loading condition. ${ }^{11)}$ In the case of a low strain rate, the overshoot appears at the starting point of the MT in the loading process followed by the upper stress plateau due to the MT. The undershoot appears at the starting point of the reverse transformation in the unloading process followed by the lower stress plateau due to the reverse transformation. In the case of the constant stress rates, the overshoot and undershoot do not appear and both slops of the stress-strain curves are steep in the stress-plateau regions due to the phase transformation during loading and unloading. The higher the stress rate is, the steeper the slope of the stress-strain curve is. In the case of a low strain rate, the phase-transformation band due to propagation of the interface between the M-phase and the parent phase progresses like Luder's band in the stressplateau region and temperature varies slightly. ${ }^{6,12)}$ In the case of the constant stress rate, many phase-transformation bands appear in the whole area of the specimen and temperature varies markedly, ${ }^{12,13)}$ resulting in the steep slope of the stressstrain curve since the MT stress varies in proportion to temperature.

3.2.2 Subloop deformation under various stress rates

The stress-strain curves obtained by the subloop loading test under the constant stress rate for the SMA wire at $T=353 \mathrm{~K}$ are shown in Fig. 3. The MT occurs in the loading process $\left(S_{M}, A_{1}\right)$. The subloop unloading process corresponds to the path $\left(A_{i}, B_{i}, C_{i}\right.$ and $\left.D_{i}\right)$. The reverse transformation 


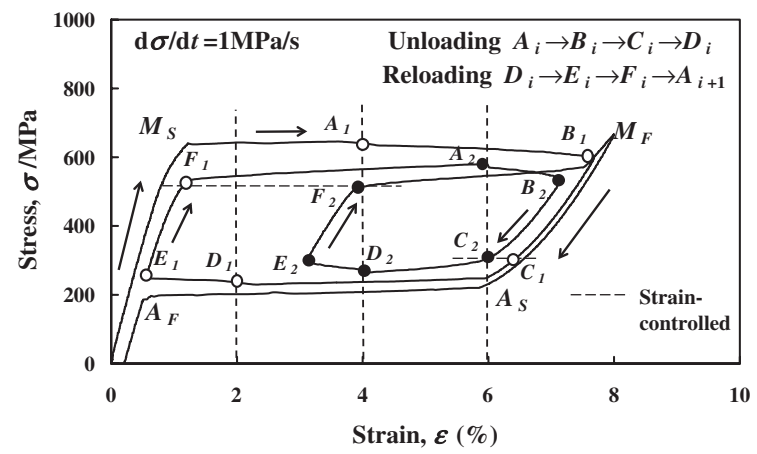

(a) $\mathrm{d} \sigma / \mathrm{d} t=1 \mathrm{MPa} / \mathrm{s}$

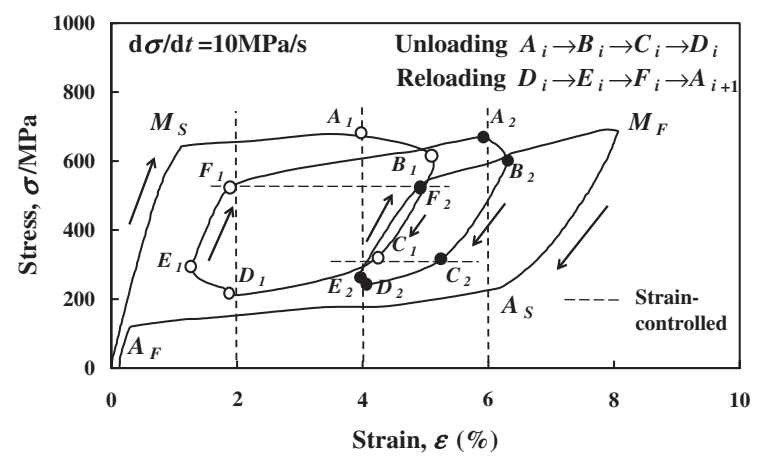

(b) $\mathrm{d} \sigma / \mathrm{d} t=10 \mathrm{MPa} / \mathrm{s}$

Fig. 3 Stress-strain curves for subloop loading under constant stress rates (a) $\mathrm{d} \sigma / \mathrm{d} t=1 \mathrm{MPa} / \mathrm{s}$, (b) $\mathrm{d} \sigma / \mathrm{d} t=10 \mathrm{MPa} / \mathrm{s}$.

occurs in the unloading process $\left(C_{i}, D_{i}\right)$. The subloop reloading process corresponds to the path $\left(D_{i}, E_{i}, F_{i}\right.$ and $\left.A_{i+1}\right)$. Strain increases in the early stage of unloading $\left(A_{i}, B_{i}\right)$ and decreases in the early stage of reloading $\left(D_{i}, E_{i}\right)$. The variation in strain is larger under a lower stress rate in both stages. These strain behaviors appear due to the following reasons. In the case of unloading $\left(A_{i}, B_{i}\right)$, temperature increases due to the MT in both the loading process $\left(S_{M}, A_{1}\right)$ and the reloading process $\left(F_{i}, A_{i+1}\right)$, but decreases during unloading $\left(A_{i}, B_{i}\right)$. The decrease in both stress and temperature during $\left(A_{i}, B_{i}\right)$ satisfies the condition of the progress for the MT, and therefore strain increases due to the MT in this stage. In the case of reloading $\left(D_{i}, E_{i}\right)$, temperature decreases due to the reverse transformation in the unloading process $\left(C_{i}, D_{i}\right)$ and increases during reloading $\left(D_{i}, E_{i}\right)$. The increase in both stress and temperature during $\left(D_{i}, E_{i}\right)$ satisfies the condition of the progress for the reverse transformation, and therefore strain decreases due to the reverse transformation in this stage. In the case of lower stress rate, the time to satisfy the condition of the progress for both transformations is longer, resulting in larger variation in strain. These strain behaviors in the subloop loading under the constant stress rate are quite different from those under a low strain rate as observed in Fig. 1. In the reloading process under a low strain rate, the stress-strain curve passes through the unloading start point $A_{i}$ as shown in Fig. 1, showing the return-point memory. However, as can be seen in Fig. 3, the reloading stress-strain curve does not pass through the unloading start point $A_{i}$. Therefore, the return-point memory does not appear under the stress-controlled condition.

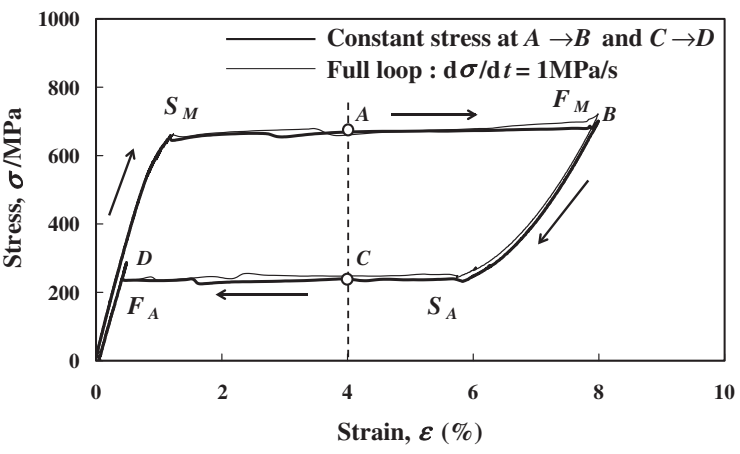

Fig. 4 Stress-strain curve for subloop loading with constant stress: $(A, B)$ and $(C, D)$.

\subsubsection{Transformation-induced creep and creep recovery under constant stress}

The stress-strain curve obtained by the subloop loading test with constant stress for the SMA wire at $T=353 \mathrm{~K}$ is shown in Fig. 4. In Fig. 4, the stress-strain curve obtained by the full loop loading under a constant stress rate of $1 \mathrm{MPa} / \mathrm{s}$ is also shown by a thin curve. The modulus of the stress rate was $1 \mathrm{MPa} / \mathrm{s}$ in the loading process $(O, A)$ and the unloading process $(B, C)$. The stress was kept constant during the processes $(A, B)$ and $(C, D)$. The condition of constant stress corresponds to a very low stress rate and the stress-strain curve is close to that of the full loop loading under the constant stress rate of $1 \mathrm{MPa} / \mathrm{s}$.

The MT occurs in the loading process $\left(S_{M}, A\right)$ and temperature increases due to the MT. In the process $(A, B)$, stress is held constant and temperature decreases under constant stress. Then the condition of the progress for the MT is satisfied and an increase in strain is induced due to the MT under constant stress. Therefore, the transformation-induced creep deformation appears. The reverse transformation occurs in the unloading process $\left(S_{A}, C\right)$ and temperature decreases. In the process $(C, D)$, stress is held constant and temperature increases under constant stress. Then the condition of the progress for the reverse transformation is satisfied and a decrease in strain is induced due to the reverse transformation under constant stress. Therefore, the transformation-induced creep recovery appears. Therefore, it is important to note that, even if the ambient temperature is constant in applications of SMAs, creep and creep recovery must appear in the stress-controlled subloop loading since the temperature of the material varies according to the phase transformation.

\subsubsection{Transformation-induced stress relaxation and stress recovery under constant strain}

The stress-strain curve obtained by the subloop loading test with constant strain for the SMA wire at $T=353 \mathrm{~K}$ is shown in Fig. 5. In Fig. 5, the stress-strain curve obtained by the full loop loading under a constant strain rate of $1.67 \times 10^{-4} \mathrm{~s}^{-1}$ is also shown by a thin curve. In the loading and unloading processes, the modulus of the stress rate was $30 \mathrm{MPa} / \mathrm{s}$. The strain was kept constant during the loading process $\left(A_{i}, B_{i}\right)$ and during the unloading process $\left(C_{i}, D_{i}\right)$. As can be seen, the stress decreases under constant strain during $\left(A_{i}, B_{i}\right)$ and increases during $\left(C_{i}, D_{i}\right)$. 


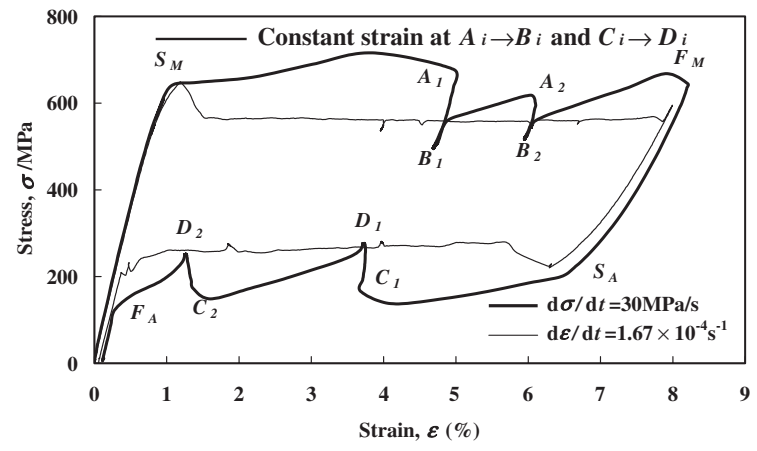

Fig. 5 Stress-strain curve for subloop loading with constant strain: $\left(A_{i}, B_{i}\right)$ and $\left(C_{i}, D_{i}\right)$.

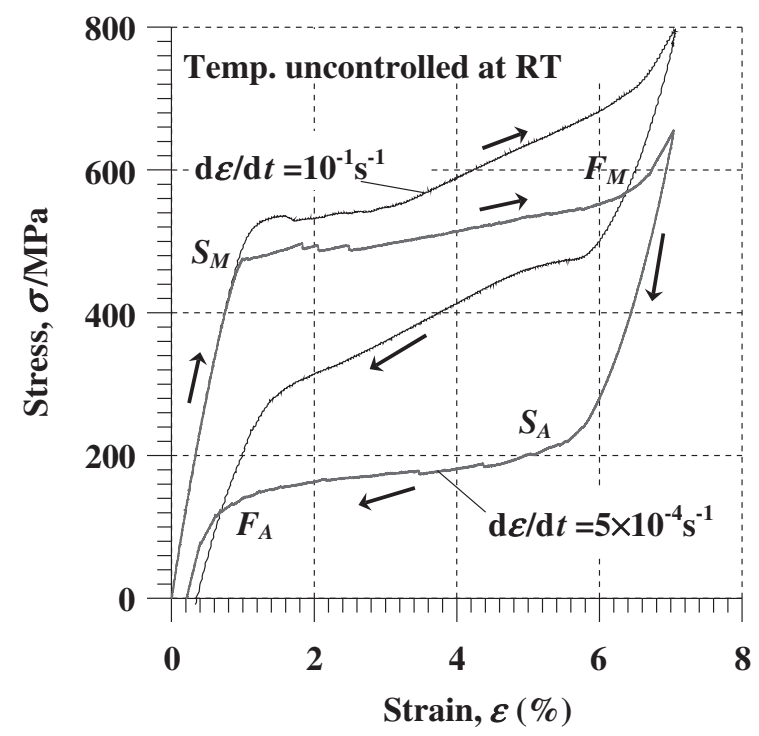

Fig. 6 Stress-strain curves at various strain rates under temperatureuncontrolled condition.

The MT occurs in the loading process $\left(S_{M}, A_{1}\right)$. In the process $\left(A_{i}, B_{i}\right)$, stress decreases under the constant strain, and the transformation-induced stress relaxation appears. The reverse transformation occurs in the unloading process $\left(S_{A}, C_{1}\right)$. In the process $\left(C_{i}, D_{i}\right)$, stress increases under the constant strain, and the transformation-induced stress recovery appears during this process. The variation in temperature of the material depends on the size of the SMA elements and the conditions of heat transfer between the SMA elements and atmosphere. Therefore, it is important to note that, even if the ambient temperature is constant in applications of SMAs, stress relaxation and stress recovery must appear in the stress-controlled subloop loading since the temperature of the material varies according to the phase transformation. ${ }^{14,15)}$

\subsection{Deformation and temperature variation under var- ious strain rates}

The stress-strain curves and variation in temperature under various strain rates are shown in Figs. 6 and 7. In Fig. 7, $\Delta T$ denotes the average temperature variation on the surface of SMA tape, and $\Delta T_{p}$ denotes the temperature variation at the point where the first transformation band with high temper-

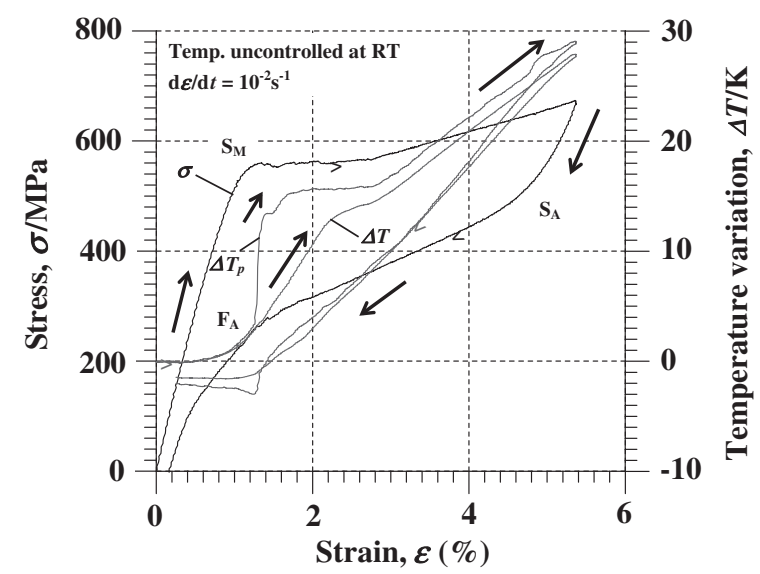

Fig. 7 Stress-strain curve and variation in temperature during loading and unloading at strain rate of $10^{-2} \mathrm{~s}^{-1}$.

ature $^{11,12)}$ like Luder's band appeared. The temperature rise due to the MT is larger under higher strain rate. ${ }^{11,12)}$ The MT stress increases in proportion to temperature. Therefore, the MT stress increases in proportion to strain rate as observed in Fig. 6. As can be seen in Fig. 7, temperature increases by $29 \mathrm{~K}$ due to the MT at strain rate of $10^{-2} \mathrm{~s}^{-1}$.

\section{Conditions for Progress of Phase Transformation and Subloop-Deformation Behavior}

In order to understand the reason why the pseudoviscoelastic behaviors of creep, stress relaxation, an increases in strain during unloading and an increases in strain during reloading appear under the stress-controlled condition, let us discuss the conditions for progress of the phase transformation and the corresponding subloop-deformation behavior.

\subsection{Conditions for progress of phase transformation in the subloop loading}

The transformation kinetics for the martensitic transformation (MT) in SMAs proposed by Tanaka et al. is expressed as follows ${ }^{16,17)}$

$$
\frac{\dot{z}}{1-z}=b_{M} C_{M} \dot{T}-b_{M} \dot{\sigma} \geq 0
$$

and for the reverse transformation

$$
-\frac{\dot{z}}{z}=b_{A} C_{A} \dot{T}-b_{A} \dot{\sigma} \geq 0
$$

where $\sigma, T$ and $z$ represent the stress, temperature and the volume fraction of the martensitic phase (M-phase), respectively. The volume fraction of the parent phase or austenitic phase (A-phase) is 1-z. An overdot denotes the time derivative. The material parameters $b_{M}, C_{M}, b_{A}$ and $C_{A}$ are determined from the experiments.

The conditions for start and finish of the MT are expressed by the following equations, respectively.

$$
\begin{aligned}
& \sigma=C_{M}\left(T-M_{s}\right) \\
& \sigma=C_{M}\left(T-M_{f}\right)
\end{aligned}
$$

The conditions for the reverse transformation are as follows, respectively. 


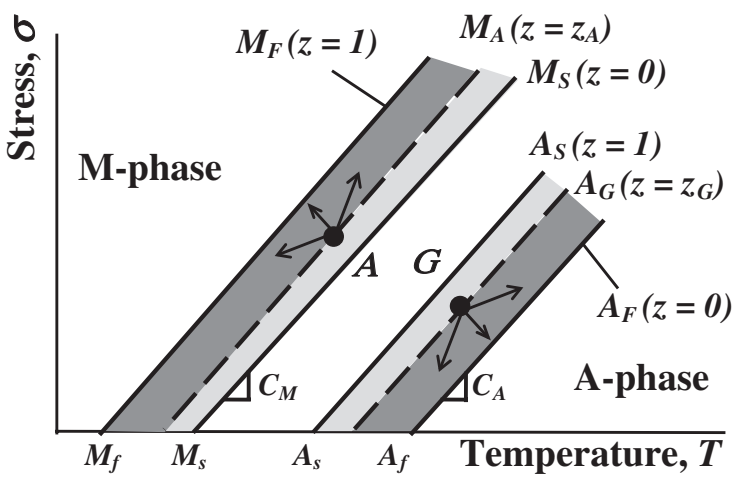

Fig. 8 Conditions for progress of the MT and the reverse transformation in the subloop loading on the stress-temperature phase diagram.

$$
\begin{aligned}
& \sigma=C_{A}\left(T-A_{s}\right) \\
& \sigma=C_{A}\left(T-A_{f}\right)
\end{aligned}
$$

The parameters $M_{s}, M_{f}, A_{s}$ and $A_{f}$ denote the start and finish temperatures for the MT and the reverse transformation under no load, respectively.

From eq. (1), the condition for progress of the MT becomes as follows since $b_{M}<0$.

$$
\begin{array}{ll}
\frac{d \sigma}{d T}>C_{M}: & \text { for } \quad d T>0 \\
\frac{d \sigma}{d T}<C_{M}: & \text { for } \quad d T<0
\end{array}
$$

From eq. (2), the condition for progress of the reverse transformation becomes as follows since $b_{A}>0$.

$$
\begin{array}{ll}
\frac{d \sigma}{d T}<C_{A}: & \text { for } \quad d T>0 \\
\frac{d \sigma}{d T}>C_{A}: & \text { for } \quad d T<0
\end{array}
$$

The conditions for progress of the phase transformation in the subloop loading during the phase transformation are shown on the stress-temperature phase diagram in Fig. 8. In Fig. 8, $M_{S}(z=0)$ and $M_{F}(z=1)$ denote the MT start and finish lines with a slope of $C_{M}$, respectively, and $A_{S}(z=1)$ and $A_{F}(z=0)$ denote the reverse transformation start and finish lines with a slope of $C_{A}$, respectively. Points $A$ and $G$ in Fig. 8 represent respectively the state of progress of the MT and the reverse transformation and the volume fractions of the M-phase at each point are $z_{A}$ and $z_{G}$. The broken lines $M_{A}$ and $A_{G}$ denote the states with the volume fractions $z_{A}$ and $z_{G}$, respectively. The conditions prescribed by eqs. (7) and (8) for progress of the phase transformation from the points $A$ and $G$ mean that stress and temperature vary in the directions shown by the arrows in Fig. 8 .

\subsection{Subloop-deformation behavior during the loading process}

The stress-strain diagram and stress-temperature path subjected to various thermomechanical subloop loadings from the point $A$ during the loading process under the stresscontrolled condition are schematically shown in Fig. 9. The stress-strain relationships (1)-(6) shown by the arrows for the subloop process from the point $A$ in Figure 9(a) correspond

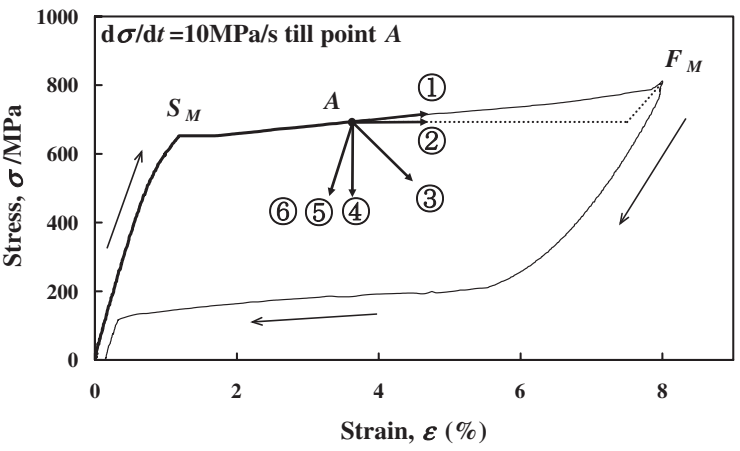

(a) Stress-strain diagram for loading paths (1)-(6)

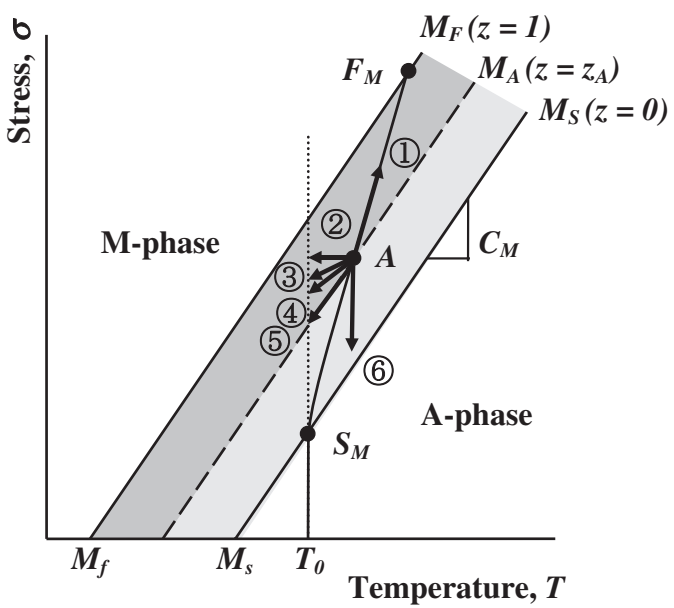

(b) Thermomechanical-subloop loading paths (1)-(6)

Fig. 9 Stress-strain diagram and various thermomechanical-subloop loading paths during the loading process under the stress-controlled condition with constant stress rate till the point $A$ (a) Stress-strain diagram for loading paths (1)-(6) (b) Thermomechanical-subloop loading paths (1)-(6).

respectively to the thermomechanical-subloop loading paths (1)-6) shown by the arrows in Fig. 9(b).

Tensile load is applied at temperature $T_{0}$ under a constant stress rate up to the point $A$. The MT starts at the point $S_{M}$ during loading. The upper stress plateau starts from the point $S_{M}$ in the stress-strain diagram and temperature increases due to the MT. The stress-strain curve and variation in temperature depend on the loading rate. ${ }^{11,12,14)}$ The thermomechanical paths (1)-(6) from the point $A$ and the corresponding deformation behaviors can be explained as follows. The path (1) corresponds to the continuous loading under a constant stress rate. In this case, the MT progresses with the condition $\mathrm{d} \sigma / \mathrm{d} T>C_{M}$ and the upper stress plateau finishes at the point $F_{M}$. The path (2) expresses the condition with holding stress constant. In this case, the MT progresses due to decrease in temperature under the constant stress and therefore creep deformation is induced due to the MT as observed in Fig. 4. The path (3) corresponds to unloading under a constant stress rate. In this case, both stress and temperature decrease with the condition $\mathrm{d} \sigma / \mathrm{d} T<C_{M}$ and the MT progresses, resulting in increase in strain. The path (4) expresses the condition with holding strain constant. In this case, the MT progresses due to decrease in temperature under the constant strain and stress relaxation is induced due to the MT as observed in Fig. 5. The path (5) represents the thermomechanical neutral load- 


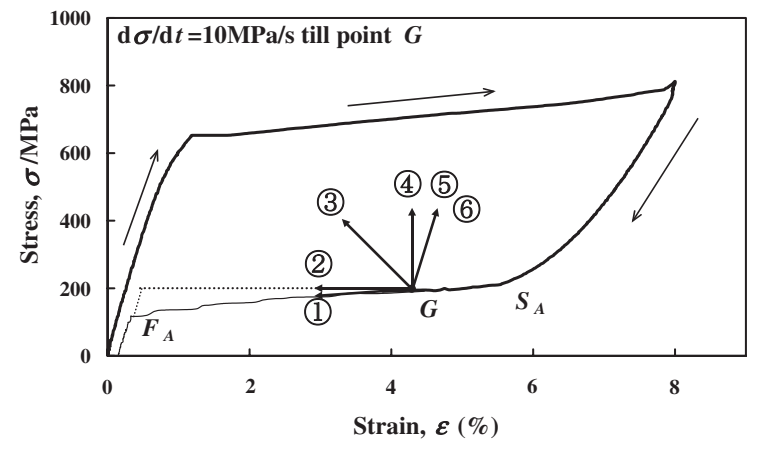

(a) Stress-strain diagram for loading paths (1)-(6)

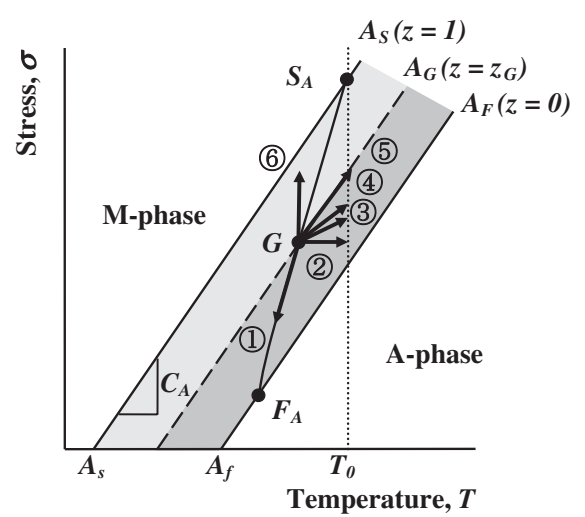

(b) Thermomechanical-subloop loading paths (1)-(6)

Fig. 10 Stress-strain diagram and various thermomechanical-subloop loading paths during the unloading process under the stress-controlled condition with constant stress rate till the point $G$ (a) Stress-strain diagram for loading paths (1-6) (b) Thermomechanical-subloop loading paths (1)-(6).

ing. ${ }^{8)}$ In this case, the MT stops with the condition $\mathrm{d} \sigma / \mathrm{d} T=C_{M}$. The path (6) is the case of elastic unloading. In this case, the MT does not appear with the condition $\mathrm{d} \sigma / \mathrm{d} T>C_{M}$.

\subsection{Subloop-deformation behavior during the unload- ing process}

The stress-strain diagram and stress-temperature path subjected to various thermomechanical subloop loadings from the point $G$ during the unloading process under the stress-controlled condition are schematically shown in Fig. 10. Tensile loading and unloading are applied continuously under a constant stress rate till the point $G$. The stressstrain relationships (1)-(6) shown by the arrows for the subloop process from the point $G$ in Fig. 10(a) correspond respectively to the thermomechanical-subloop loading paths (1)-(6) shown by the arrows in Fig. 10(b).

The reverse transformation starts at the point $S_{A}$ during unloading at temperature $T_{0}$. The lower stress plateau starts from the point $S_{A}$ in the stress-strain diagram and temperature decreases due to the reverse transformation. The paths (1)-(6) from the point $G$ and the corresponding deformation behaviors can be explained as follows. The path (1) corresponds to the continuous unloading under a constant stress rate. In this case, the reverse transformation progresses with $\mathrm{d} \sigma / \mathrm{d} T>C_{A}$ and the lower stress plateau finishes at the point $F_{A}$. The path (2) expresses the condition with holding stress constant. In this case, the reverse transformation progresses with increasing temperature under the constant stress and creep recovery is induced due to the reverse transformation as observed in Fig. 4. The path (3) corresponds to reloading under a constant stress rate. In this case, both stress and temperature increases with $\mathrm{d} \sigma / \mathrm{d} T<C_{A}$ and the reverse transformation progresses, resulting in decrease in strain. The path (4) expresses the condition with holding strain constant. In this case, the reverse transformation progresses with increasing temperature under the constant strain and stress recovery is induced due to the reverse transformation as observed in Fig. 5. The path (5) represents the thermomechanical neutral reloading. In this case, $\mathrm{d} \sigma / \mathrm{d} T=C_{A}$ and the reverse transformation stops. The path (6) is the case of elastic reloading. In this case $\mathrm{d} \sigma / \mathrm{d} T>C_{A}$ and the reverse transformation does not appear.

\section{Neutral Loading Behavior in the Subloop}

In order to perform the thermomechanical test including the neutral loading, it is necessary to keep the condition: $\mathrm{d} \sigma / \mathrm{d} T=C_{M}$. Therefore, the stress rate and the temperature rate must satisfy the condition: $\mathrm{d} \sigma / \mathrm{d} t=C_{M} \times \mathrm{d} T / \mathrm{d} t$. In the experimental apparatus, the precise temperature control of the specimen is difficult since temperature of the specimen varies due to the MT and the reverse transformation depending on the loading rate. Therefore, it is very difficult to achieve the neutral loading condition precisely. We tried to achieve this condition by many experiments and finally could carry out the following test. The controlled-stress variation in the test is shown by the solid line in Fig. 11(b). At first, uniaxial tensile load was applied to the SMA tape up to stress of $500 \mathrm{MPa}$ (point $A$ ) under a constant stress rate of $12.5 \mathrm{MPa} / \mathrm{s}$ followed by unloading under a decreasing stress rate of $0.56 \mathrm{MPa} / \mathrm{s}$ for $180 \mathrm{~s}$ in $(A, D)$. Following the unloading process $(A, D)$, reloading $(D, G)$ and perfect unloading $(G, H)$ were performed under a constant stress rate of $12.5 \mathrm{MPa} / \mathrm{s}$.

The stress-strain curve and variation in temperature obtained by the thermomechanical test including the neutral loading for the SMA tape at $293 \mathrm{~K}$ are shown by the solid line and the dotted line in Fig. 11, respectively. The corresponding thermomechanical loading path in the loading process is schematically shown in Fig. 12. As can be seen in Figs. 11 and 12, the MT starts at the point $S$ and progresses till the point $A$. Temperature increases from the point $S$ till the point $A$ due to the MT. During unloading $(A, B)$, temperature decreases and strain increases due to the MT, corresponding to the path (3) in Fig. 9. The neutral unloading condition is achieved in the process $(B, C)$. In this process, the ratio of variation in stress to that in temperature is as follows.

$$
\frac{\Delta \sigma}{\Delta T}=\frac{-20 \mathrm{MPa}}{-3 \mathrm{~K}}=6.7 \mathrm{MPa} / \mathrm{K}
$$

The ratio of $\Delta \sigma / \Delta T$ is close to a value of $6 \mathrm{MPa} / \mathrm{K}$ which is the slope of the transformation line $C_{M}$ of the material. Therefore, the neutral unloading condition is satisfied, resulting in stop of the MT. In the processes $(C, D)$ and $(D, E)$, temperature varies slightly, and therefore elastic unloading and reloading occur, respectively. In the process $(E, F)$, the MT grows again. 


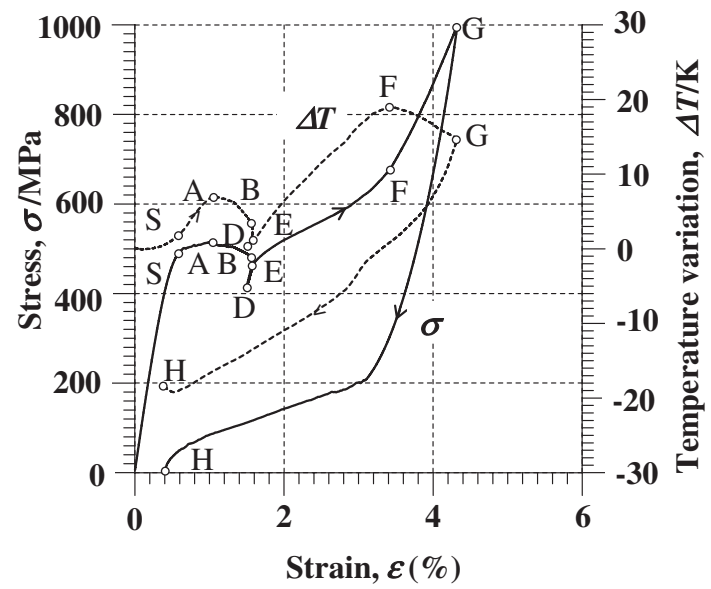

(a) Stress-strain curve and variation in temperature

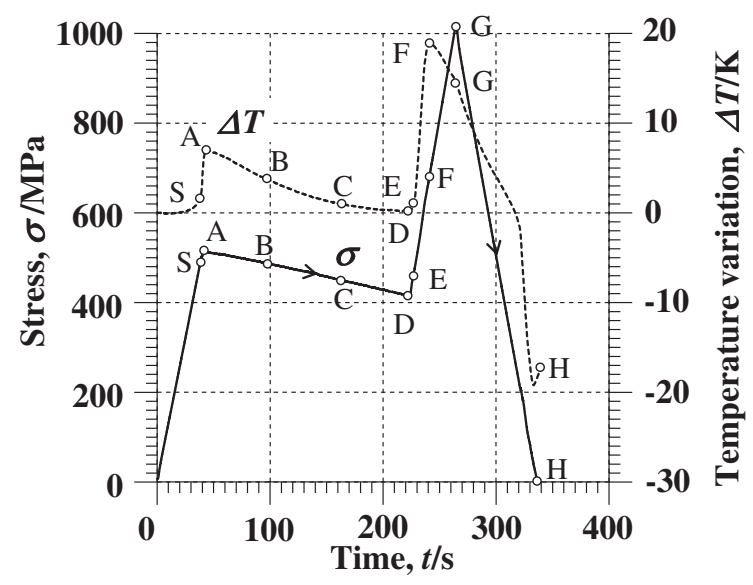

(b) Variations in stress and temperature with time

Fig. 11 Stress-strain diagram and variation in stress and temperature with time obtained by the thermomechanical test with two kinds of stress rate including the neutral unloading (a) Stress-strain curve and variation in temperature (b) Variations in stress and temperature with time.

In the process $(A, B)$, the observed decrease in temperature is almost the same as that in the process $(B, C)$. This must occur due to the following reason. The temperature was measured on the surface of the central part (length of $60 \mathrm{~mm}$ ) of the specimen. The gauge length to measure strain is $100 \mathrm{~mm}$. The grippers of the specimen are quite larger than the specimen. The heat generated due to the MT in the specimen transfers quickly into the grippers. Therefore, temperature in the neighborhood of the grippers can decrease significantly during unloading, and the MT can progress in this part. As the result, the observed temperature in the process $(A, B)$ at the central part of the specimen does not decrease markedly as much as estimated.

\section{Conclusions}

The subloop deformation behavior under the stresscontrolled condition was investigated for TiNi SMA. The influence of loading condition on the deformation behavior was discussed based on the experimental results and theoretical consideration. The results obtained can be summarized as follows.

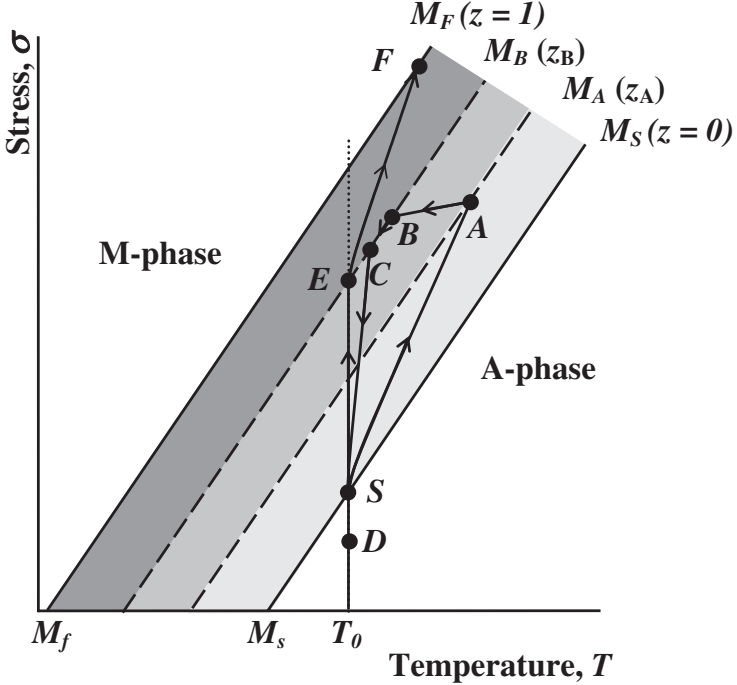

Fig. 12 Thermomechanical loading path on the stress-temperature phase diagram for the thermomechanical test including the neutral loading (SA: path (1), $\mathrm{AB}$ : path (3), BC: path (5), $\mathrm{CD}$ : path (6), DE: elastic reloading, EF: path (1) in Fig. 3).

(1) In the case of low strain rate for the subloop loading, the return-point memory appears and therefore the correspondence between stress and strain can be obtained. If deformation rate is low, the working stroke and the driving force can be controlled for SMA elements.

(2) In the case of the stress-controlled condition for the subloop loading, the return-point memory dose not appears. Even if the ambient temperature is constant, temperatures of SMA varies due to the MT. Based on the variation in temperature, strain increases during unloading and decreases during reloading. In addition, the transformation-induced creep and stress relaxation can appear under the stresscontrolled condition even if the ambient temperature is constant. In order to perform the precise control of the working stroke and the driving force of SMA elements, it is necessary to take account of this property.

(3) If the ratio of variation in stress to variation in temperature is equal to the slope of the transformation line, the neutral loading condition is satisfied for the subloop loading and the MT stops. If this condition is satisfied, only the elastic deformation occurs in SMA elements.

\section{Acknowledgement}

The experimental work was carried out with the assistance of students of Aichi Institute of Technology, to whom the authors wish to express their gratitude. The important point of this study was suggested by the discussion with Professor Bogdan Raniecki at IFTR of Polish Academy of Sciences, to whom the authors wish to express their gratefulness. The authors also wish to extend their thanks to the Joint Research Project between two countries supported by Polish Academy of Sciences and Japan Society for Promotion of Sciences (JSPS), the Postdoctoral Fellowship supported by JSPS and the Basic Research (C) of a Grant-in-Aid of Scientific Research supported by JSPS for their financial supports. 


\section{REFERENCES}

1) H. Funakubo (ed.): Shape Memory Alloys, (Gordon and Breach Science Pub., 1987) pp. 1-60.

2) T. W. Duering, K. N. Melton, D. Stokel and C. M. Wayman, (eds.): Engineering Aspects of Shape Memory Alloy, (Butterworth-Heinemann, 1990) pp. 1-35.

3) K. Otsuka and C. M. Wayman, (eds.): Shape Memory Materials, (Cambridge University Press, 1998) pp. 1-49.

4) T. Saburi, (ed.): Shape Memory Materials (Trans Tech Pub., 2000) pp. 315-366.

5) Y. Y. Chu and L. C. Zhao (eds.): Shape Memory Materials and Its Applications, (Trans Tech Pub., 2002) pp. 177-284.

6) J. S. Shaw and S. Kyriakides: J. Mech. Phys. Solids 43 (1995) 12431281.

7) P. H. Lin, H. Tobushi, K. Tanaka, T. Hattori and M. Makita: J. Intell. Mat. Syst. Struct. 5 (1994) 694-701.

8) B. Raniecki, C. Lexcellent and K. Tanaka: Arch. Mech. 44 (1992) 261-
284.

9) G. Socha, B. Raniecki and S. Miyazaki: 33rd Solid Mechanics Conference, (2000) pp. 369-370.

10) H. Tobushi, H. Iwanaga, K. Tanaka, T. Hori and T. Sawada: Continuum Mech. Thermodyn. 3 (2000) 79-93.

11) H. Tobushi, K. Tanaka, Y. Shimeno, W. K. Nowacki and S. P. Gadaj: Proc. Instn. Mech. Engrs., Part L, J. Materials: Design and Applications 213 (1999) 93-102.

12) E. A. Pieczyska, H. Tobushi, S. P. Gadaj and W. K. Nowacki: Mater. Trans. 47 (2006) 670-676.

13) E. A. Pieczyska, S. P. Gadaj, W. K. Nowacki and H. Tobushi: Exp. Mech. 46 (2006) 531-542.

14) S. P. Gadaj, W. K. Nowacki and H. Tobushi: Arch. Mech. 51 (1999) 649-663.

15) E. A. Pieczyska, S. P. Gadaj, W. K. Nowacki and H. Tobushi: Int. J. Applied Electromagnetics Mechanics 23 (2006) 3-8.

16) K. Tanaka: Res. Mechanica 18 (1986) 251-263.

17) K. Tanaka, S. Kobayashi and Y. Sato: Int. J. Plasticity 2 (1986) 59-72. 